New windows on massive stars: asteroseismology, interferometry, and spec-

tropolarimetry

Proceedings IAU Symposium No. 307, 2014

G. Meynet, C. Georgy, J. H. Groh \& Ph. Stee, eds.

(C) International Astronomical Union 2015 doi:10.1017/S1743921314006565

\title{
Herschel/PACS: Constraining clumping in the intermediate wind region of $\mathrm{OB}$ stars
}

\author{
M. M. Rubio-Díez ${ }^{1}$, F. Najarro ${ }^{1}$, J. O. Sundqvist ${ }^{2}$, A.Traficante ${ }^{3}$, \\ J. Puls ${ }^{2}$, L. Calzoletti ${ }^{4,5}$, A. Herrero ${ }^{6,7}$, D. Figer ${ }^{8}$ and \\ J. Martin-Pintado ${ }^{1}$ \\ ${ }^{1}$ Centro de Astrobiología, CSIC-INTA, Madrid, Spain \\ email: mmrd@cab.inta-csic.es \\ ${ }^{2}$ Universitätssternwarte München, München, Germany \\ ${ }^{3}$ Jodrell Bank Centre for Astrophysics, School of Physics and Astronomy, University of \\ Manchester, Manchester, UK \\ ${ }^{4}$ ASI Science Data Center, Roma, Italy \\ ${ }^{5}$ INAF, Osservatorio Astronomico di Roma, Rome, Italy \\ ${ }^{6}$ Instituto de Astrofísica de Canarias, Tenerife, Spain \\ ${ }^{7}$ Dpto. de Astrofísica, Universidad de La Laguna, Tenerife, Spain \\ ${ }^{8}$ Rochester Institute of Technology, NY, USA
}

\begin{abstract}
At present, it is well established that previously accepted mass-loss rates $(\dot{M})$ of luminous OB stars may be overestimated when clumping is neglected. Our Herschel/PACS Far-Infrared (Far-IR) observations of a set of OB stars allow us to improve our knowledge of clumping stratification, constraining clumping properties in intermediate wind regions. In this work, better sampled clumping structure estimates are provided for $\iota$ Ori, $\epsilon$ Ori and $\xi$ Per as well as an initial estimate of the clumping properties of the wind from $\tau$ Sco. These observations will allow us to obtain reliable mass-loss rates and improve our understanding of the wind physics.
\end{abstract}

\section{Introduction}

It is currently accepted that stellar winds of massive stars are not smooth, but show small scale inhomogeneities. Such "wind clumps" have a crucial effect on the standard mass-loss rate $(\dot{M})$ diagnostics: for a given mass loss, microclumping (optically thin clumps) increases emission that depends on $\rho^{2}$ (H emission, IR/radio free-free emission) while having no effect on processes that depend on $\rho$ (UV resonance lines absorption). On the other hand, macroclumping (optically thick clumps) leads to higher $\dot{M}$ estimates if derived from resonance lines. Not taking into account clumping results in inconsistencies between different mass-loss diagnostics and overestimated $\dot{M}$ (Fullerton et al. 2006; Sundqvist et al. 2010). Therefore, a reliable knowledge of the clumping quantified by the clumping factor $\left(f_{\mathrm{cl}}=f_{v}^{-1} \equiv<\rho^{2}>/<\rho>^{2}\right.$, Owocki et al. 1988) and its radial stratification (Sundqvist \& Owocki 2013) is crucial to constrain the "true" $\dot{M}$ of OB stars. We argue that only a consistent treatment of ALL possible diagnostics, scanning different parts of the winds, and analyzed by means of state of the art model atmospheres, will enable us to constrain clumping through the whole wind and $\dot{M}$ itself. To this end, we have assembled a variety of multi-wavelength data (from optical to radio) of a carefully selected sample of 28 O4-B8 stars, including new Far-IR diagnostics of free-free emission. We have used our photometric Herschel/PACS observations at 70, 100 and $160 \mu \mathrm{m}$ (programs: OT1/OT2_mrubio), which uniquely constrain the clumping properties of the intermediate wind regions, to derive the clumping properties of the entire outflow. 

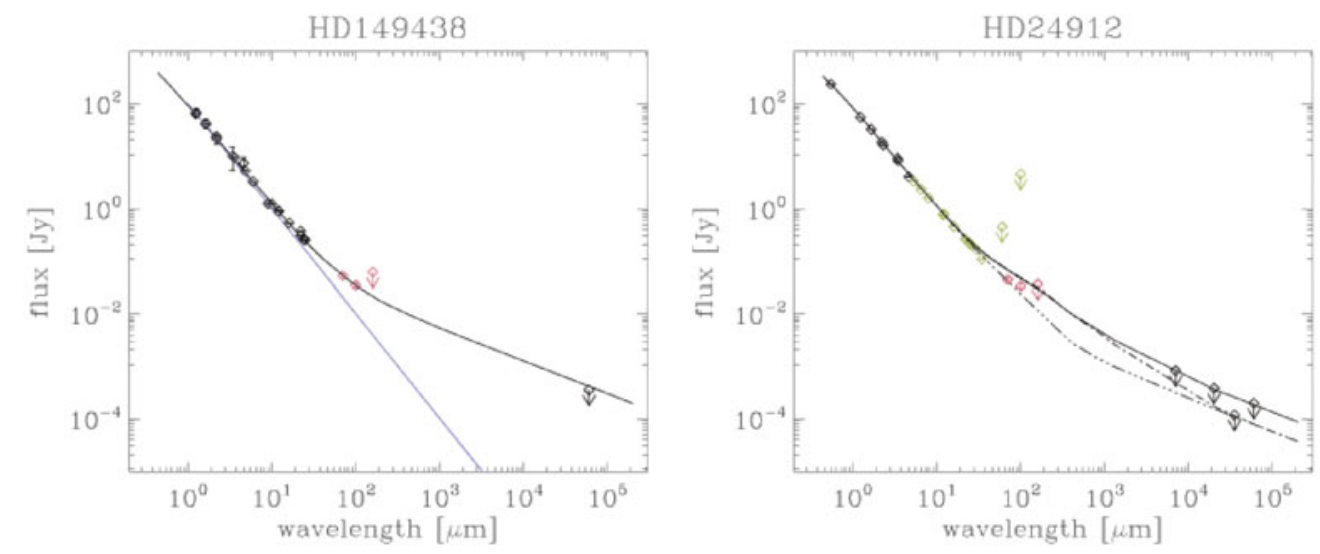

Figure 1. Different computed SED models including microclumping structure of the wind built from IR/radio continuum archival measurements (black and green symbols) and PACS fluxes at 70,100 and $160 \mu \mathrm{m}$ (red symbols). Arrows denote upper limits. Left: $\tau$ Sco PACS flux values agree with the new unclumped model, $f_{\mathrm{cl}}=1$, computed for $\dot{M}=3.23 \times 10^{-7} M_{\odot} \mathrm{yr}^{-1}$ (black solid line). The blue line represents $\tau$ Sco SED for spectral index $\alpha=2$. Right: $\xi$ Per. Clumping models by Puls et al. (2006); Solid line: $\dot{M}=2.3 \times 10^{-6} M_{\odot} \mathrm{yr}^{-1}$ and $f_{\text {clin }}=2.1, f_{\text {clmid }}=5$, $f_{\text {clout }}=1$; dotted-dashed line: $\dot{M}=1.2 \times 10^{-6} M_{\odot} \mathrm{yr}^{-1}, f_{\text {clin }}=8, f_{\text {clmid }}=20, f_{\text {clout }}=1$; dashed-triple dotted line: $\dot{M}=1.2 \times 10^{-6} M_{\odot} \mathrm{yr}^{-1}, f_{\text {clin }}=8, f_{\text {clmid }}=f_{\text {clout }}=1$. PACS data break the degeneracy between models pointing to lower mass-loss and clumping structure in the intermediate wind region.

\section{Remarks}

In a first step, we test previous clumping simulations for $\iota$ Ori, $\epsilon$ Ori, $\xi$ Per (Puls et al. 2006 and Najarro et al. 2011) against new Herschel/PACS observations at 70, 100 and $160 \mu \mathrm{m}$. These results together with the first clumping structure study for $\tau$ Sco and previously analyzed objects (Rubio-Díez et al. 2013: RD13) make a total subsample of 11 stars (8 O giant/supergiant stars and $3 \mathrm{~B}$ supergiant/dwarf stars). When this subsample is considered, we find: i) Far-IR observations disentangle the uncertainty in the previous clumping models of $4 \mathrm{O}$ stars of our sample and point to low clumping in the intermediate wind region (e.g.: $\xi$ Per, Fig. 1 right; $\lambda^{1}$ Ori and $\lambda$ Cep, RD13); ii) for 2 O stars, our PACS flux measurements confirm the clumping structure derived in previous work (e.g. $\zeta$ Puppis, $\alpha$ Cam, see RD13); iii) the only PACS detection of $\iota$ Ori at $70 \mu \mathrm{m}$ shows this object as a Rayleigh-Jeans emitter; iv) for the two early-B stars analyzed, the measurements at Far-IR wavelengths indicate slight excess emission ( $\tau$ Sco, Fig. 1 left) or a stronger clumped wind than predicted $(\epsilon$ Ori $) ; \mathbf{v})$ the uncertainty in the Far-IR flux measurements of CygOB2\#7 and \#11, due to their complex surroundings (visible in PACS maps), prevents from drawing conclusions about their clumping structure at the intermediate wind region without a parallel spectroscopic analysis. All the sample stars will be subject to a second-stage analysis: a spectroscopic multi-wavelength study with the CMFGEN code (Hillier \& Miller 1998). This second phase is mandatory in order to check that the derived clumping properties are consistent with the observed spectral lines, and will provide further insight into the wind physics and mass-loss rates.

\section{References}

Fullerton, A. W., Massa, D. L., \& Prinja, R. K. 2006, ApJ 637, 1025

Hillier, D. J. \& Miller, D. L. 1998, ApJ 496, 407

Najarro, F., Hanson, M. M., \& Puls, J. 2011, A $\& A$ 535, A32 
Herschel/PACS: Constraining clumping in the intermediate wind region of OB stars 139

Owocki, S. P., Castor, J. I., \& Rybicki, G. B. 1988, ApJ 335, 914

Puls, J., Markova, N., Scuderi, S., et al. 2006, A\& A 454, 625

Rubio-Díez, M. M., Najarro, F., Traficante, A., et al. 2013, in Massive Stars: From alpha to Omega

Sundqvist, J. O. \& Owocki, S. P. 2013, MNRAS 428, 1837

Sundqvist, J. O., Puls, J., \& Feldmeier, A. 2010, A\&A 510, A11

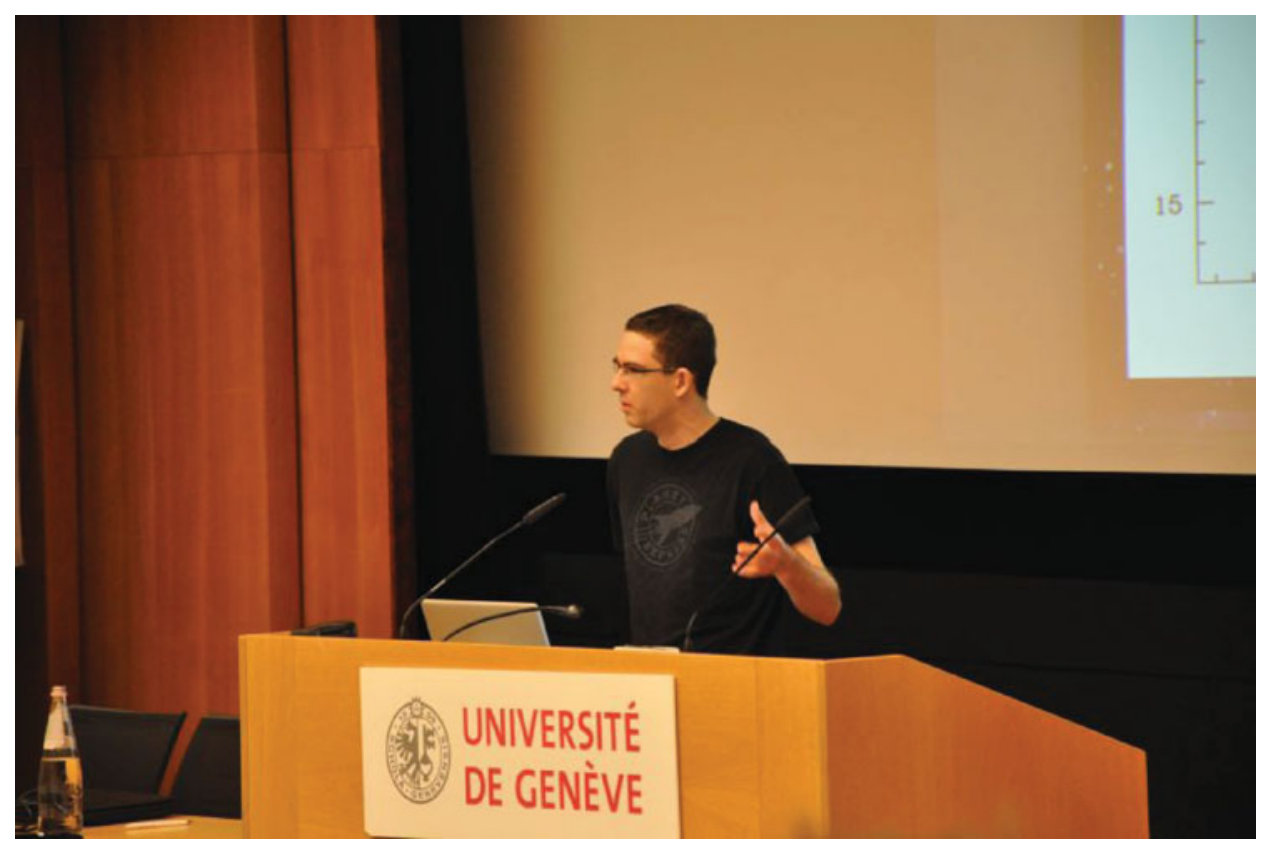

William Chantereau

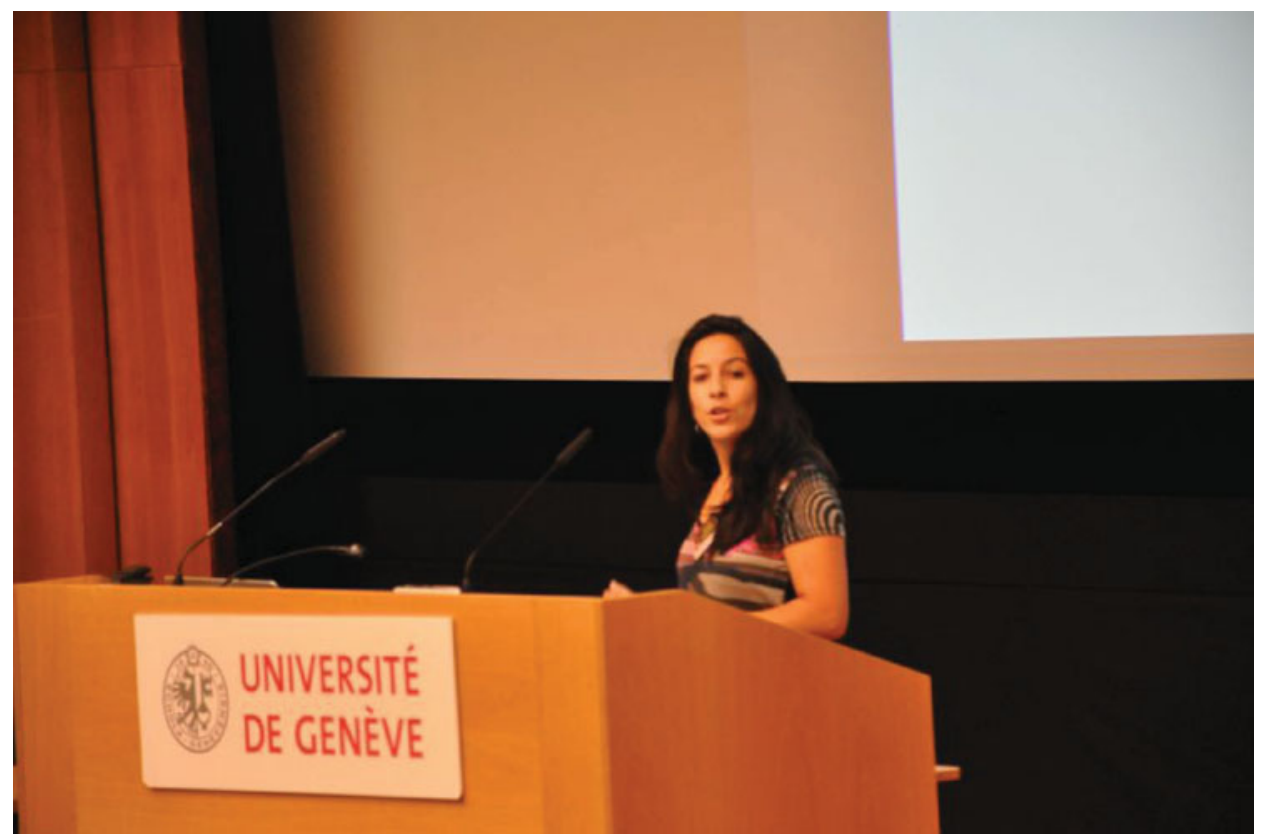

Anahí Granada 